\title{
Suppressive Effect of a Thioamide-Related Compound SH-2251 on a Murine Allergic Rhinitis Model
}

Takashi Kitani $^{1}$, Saho Maruyama ${ }^{2}$, Kunihide Aoishi ${ }^{1}$, Naoya Nishida ${ }^{1}$, Hideo Ogawa ${ }^{1}$, Yasunori Abe ${ }^{1}$, Yuji Hayashi ${ }^{1}$, Masakatsu Yamashita ${ }^{2}$ and Naohito Hato ${ }^{{ }^{*}}$ ${ }^{1}$ Department of Otorhinolaryngology Head and Neck Surgery, Graduate School of Medicine, Ehime University, Toon, Ehime, Japan

${ }^{2}$ Department of Immunology, Graduate School of Medicine, Ehime University, Toon, Ehime, Japan

"Corresponding author: Naohito Hato, Department of Otorhinolaryngology Head and Neck Surgery, Graduate School of Medicine, Ehime University, Shitsukawa, Toon city, Ehime, Japan, Tel: +81-89-960-5366; Fax: +81-89-960-5368; E-mail: nhato@m.ehime-u.ac.jp

Received date: October 10, 2017; Accepted date: October 16, 2017; Published date: October 23, 2017

Copyright: @ 2017 Kitani T, et al. This is an open-access article distributed under the terms of the Creative Commons Attribution License, which permits unrestricted use, distribution, and reproduction in any medium, provided the original author and source are credited.

\begin{abstract}
Background: SH-2251 inhibits IL-5 production and type2 airway inflammation in a murine bronchial asthma model. However, the therapeutic effect of SH-2251 on allergic rhinitis (AR) has not been tested. We investigated whether or not SH-2251 could improve the nasal symptoms and inflammation in the nasal mucosa of a murine model of AR.
\end{abstract}

Methods: AR was induced via intraperitoneal injection of ovalbumin (OVA) followed by daily intranasal challenge with OVA. SH-2251 $(10 \mathrm{mg} / \mathrm{kg}$ ) or vehicle (DMSO+Tween80) was administered orally once a day in the same period as the nasal challenge. Nasal symptoms were evaluated by counting the number of sneezing and nasal rubbing events on days $14,17,21,28$ and 35 . On day 35 , the levels of $\|4\| 5,, \| 13$, Ifny and II1rl1 mRNA in the nasal mucosa were examined. The histological findings were examined on day 36 .

Results: The numbers of sneezing and nasal rubbing events were significantly decreased by the administration of SH-2251. Both eosinophil infiltration and the hickness of the nasal mucosa were improved in the SH-2251administered mice compared that in vehicle-treated control mice. The mRNA expression of II5, II13 and II1rl1 in the nasal mucosa was significantly decreased in the $\mathrm{SH}-2251$-treated group compared to the vehicle group.

Conclusion: These results suggest that SH-2251 may be a new therapeutic candidate for AR. In addition, $\mathrm{SH}-2251$ might improve the pathogenesis of type2 chronic inflammation by targeting the IL-33 signaling via the inhibition of II1rl1 expression.

Keywords: Allergic rhinitis; SH-2251; Th2 cell; Eoshinophil; IL-5; IL-1rl1

\section{Introduction}

Allergic rhinitis (AR) affects $10 \%$ to $40 \%$ of the world's population [1]. AR generally indicates nasal mucosal inflammation characterized by IgE-mediated allergic immune responses [2]. Histologically, the type of nasal mucosal inflammation is exudative inflammation. Suppurative and allergic inflammation is particularly common. Various medications have been developed to prevent or reduce the symptom of AR such as corticosteroids, antihistamines, decongestants, anticholinergics and leukotriene receptor antagonists [3]. However, permanent curative medicines for AR have not been established yet.

Functionally distinct Th subsets have been reported, including Th1, Th2, Th17 and inducible regulatory T (iTreg) cells [4-7]. The abnormal activation of Th2 cells is responsible for allergic inflammatory diseases, including AR [8]. Inhaled aeroallergens are captured and processed by antigen-presenting cells and presented to Th2 cells [2]. Th2 cells can stimulate several type 2 effector responses that are hallmarks of AR; specifically, IL-4 can promote B cell isotype class-switching to IgE. IL-5 can promote the recruitment of eosinophils, and IL-13 can stimulate mucus hyper-secretion and airway hyper-reactivity [9].
SH-2251, a thioamide-related compound, was originally synthesized as an inhibitor for IL-5 production. Suzuki et al. reported that SH-2251 inhibits the generation of IL-5-producing Th2 cells in vitro [10]. Furthermore, Th2-dependent airway inflammation was attenuated by the administration of SH-2251 in a murine model of OVA-induced bronchial asthma [10]. Gfil, a transcriptional repressor, is required for the induction of active histone marks on the $\mathrm{Il} 5$ gene locus and subsequent differentiation of IL-5-producing Th2 cells [11]. We found that the therapeutic effect of SH-2251 was mediated, in part, by inhibiting Gfil expression.

We investigated the therapeutic effect of SH-2251 administration on murine OVA-induced AR.

\section{Materials and Methods}

\section{Animals}

$\mathrm{BALB} / \mathrm{c}$ female mice were purchased from Charles River Laboratories Japan, Inc. (Kanagawa, Japan). All mice were maintained in an air-conditioned room with controlled temperature $\left(24^{\circ} \mathrm{C}\right)$ and used at 6-8 weeks of age. All procedures were conducted in accordance with the Guidelines for Animal Experiments at Ehime University. 
Citation: Kitani T, Maruyama S, Aoishi K, Nishida N, Ogawa H, et al. (2017) Suppressive Effect of a Thioamide-Related Compound SH-2251 on a Murine Allergic Rhinitis Model. J Allergy Ther 8: 267. doi:10.4172/2155-6121.1000267

Page 2 of 5

\section{Sensitization, challenge with ovalbumin (OVA) and administration of SH-2251}

$\mathrm{BALB} / \mathrm{c}$ mice were sensitized intraperitneally with $20 \mu \mathrm{g}$ of OVA containing $1 \mathrm{mg}$ of alum as adjuvant on days 0,7 and 14 . From day 14 , the mice were challenged intranasally with $200 \mu \mathrm{g}$ OVA in $20 \mu \mathrm{l}$ PBS twice a day for 3 weeks. Following sensitization and nasal challenge in 3 weeks, the mice exhibited typical symptoms of AR such as sneezing and nasal rubbing when exposed to OVA. Sensitization was confirmed by measuring the serum OVA-specific IgE.

SH-2251 (United States Patent No. US 7632865 B2) was synthesized and provided by Ishihara Sangyo Kaisha, Ltd. The purity of the SH-2251 used in the experiments was $99.1 \%$. SH-2251 $(10 \mathrm{mg} / \mathrm{kg})$ or Vehicle (DMSO+Tween 80 ) was administered orally once a day from days 14 to day 35 .

\section{The evaluation of nasal symptoms}

Nasal symptoms were evaluated by counting the numbers of sneezing and nasal rubbing events in $10 \mathrm{~min}$ from $10 \mathrm{~min}$ after the last nasal challenge. Nasal symptoms were evaluated on days 14, 17, 21, 28 and 35 .

\section{Histology}

At $24 \mathrm{~h}$ after the final nasal challenge, mice were anesthetized with ketamine hydrochloride and xylazine hydrochloride. Mice were perfused through the left ventricle of the heart with PBS followed by $4 \%$ paraformaldehyde and then their heads were removed. They were fixed with $4 \%$ paraformaldehyde overnight at $4^{\circ} \mathrm{C}$ and decalcified with $0.5 \mathrm{M}$ EDTA solution ( $\mathrm{pH}$ 7.5) for 3 weeks. After dehydration with ethanol, the samples were embedded in paraffin. The embedded samples were cut into $3-\mu \mathrm{m}$-thin coronal sections by a microtome and rinsed in d-limonene and ethanol for the removal of paraffin. The frontal sections of the nose, including the respiratory mucosa, were stained with hematoxylin and eosin (H \& E) to evaluate the number of eosinophils and the thickness of the nasal mucosa. Giemsa staining was also applied to evaluate the eosinophil infiltration of nasal mucosa. The thickness of the nasal mucosa was measured using the DP controller software program, version 03.02 (Olympus, Tokyo, Japan) as previously described [12].

\section{Th2 cell differentiation in vitro}

Naïve CD4 T (CD44 low CD62L high CD25 negative) cells were prepared using a $\mathrm{CD} 4+\mathrm{CD} 62 \mathrm{~L}+\mathrm{T}$ cell isolation kit II (cat\#130-093-227; Miltenyi Biotec San Diego, CA, USA). Naïve CD4 T cells $\left(1.5 \times 10^{6}\right.$ cells $)$ were stimulated with an immobilized anti-TCR- $\beta$ $\mathrm{mAb}(3 \mu \mathrm{g} / \mathrm{ml}$, H57-597; Bio Legend, San Diego, CA, USA) and an anti-CD28 mAb $(1 \mu \mathrm{g} / \mathrm{ml}, 37.5$; Bio Legend) for two days under the conditions indicated. Next, the cells were transferred to a new plate and further cultured in the presence of cytokines for five days. The cytokine conditions were as follows: IL-2 $(10 \mathrm{ng} / \mathrm{ml})$, IL-4 $(1 \mathrm{ng} / \mathrm{ml}, 3$ $\mathrm{ng} / \mathrm{ml})$ and anti- IFN- $\gamma \mathrm{mAb}(5 \mu \mathrm{g} / \mathrm{ml}, \mathrm{R} 4-6 \mathrm{~A} 2$; Bio Legend).

\section{Total RNA preparation}

One hour after the final nasal challenge, the mice were sacrificed by cervical dislocation and their heads were removed. They were cut sagittally at almost the center and the mucosa of the nasal septum, the roof of the nose, turbinate and the lateral walls were obtained. Total RNA was isolated using the TRI Reagent (Molecular Research Center, Inc., Cincinnati, OH, USA).

\section{Quantitative reverse transcription polymerase chain reaction (qRT-PCR)}

cDNA was synthesized using the Superscript VILO cDNA synthesis kit (cat\#11754; Thermo Fisher Scientific, Waltham, MA, USA). Quantitative RT-PCR was performed as previously described (10), using StepOnePlus Real-Time PCR System (Thermo Fisher Scientific, Waktham, MA).

The specific primers and Roche Universal probes used in qRT-PCR were as follows:

18s: 5' GCAATTATTCCCCATGAACG' (forward), 5' GGGACTTAATCAACGCAAGC 3' (reverse), probe \#48

Il4: 5' CCTGCTCTTCTTTCTC 3' (forward), 5' CACATCCATCTCCGTG 3' (reverse), probe \#92

Il13: 5' CGTTGCACAGGGGAGTCT 3' (forward), 5' CCTCTGACCCTTAAGGAGCTTAT 3' (reverse), probe \#17

Ifny: 5' ATCTGGAGGAACTGGCAAA 3' (forward), 5' TTCAAGACTTCAAAGAGTCTGAGGTA 3' (reverse), probe \#21

Il1rl1: 5' GGCCAGAACAAACACCTGTC 3' (forward), 5' GACCTGTTACCTGGGCAAGAT 3' (reverse), probe \#31

Il1 rap: 5' CATCCATCTGGTCGCT 3' (forward), 5' AAATACCACAGAAGTC 3' (reverse), probe \#3

Il5 (\#17621 C7; Applied Bio systems)

\section{Statistical analyses}

Student's t-test was performed for the statistical analyses. Differences with P-value of less than 0.05 were considered significant.

\section{Results}

\section{Nasal symptoms of AR were attenuated by SH-2251 administration}

A schematic representation of experimental schedule is shown in Figure 1 . In brief, mice were sensitized intraperitoneally with OVA on days 0,7 and 14. From day 14, the mice were challenged intranasally with OVA twice a day for 3 weeks. Although no sneezing was observed on days 14 and 17, the mice exhibited typical symptoms of AR, such as sneezing and nasal rubbing when exposed to OVA after 3 weeks nasal OVA challenge (Figure 2). The numbers of sneezing (Figure 2A) and nasal rubbing (Figure 2B) events were significantly reduced by $\mathrm{SH}-2251$ administration compared to vehicle-treated control mice. 


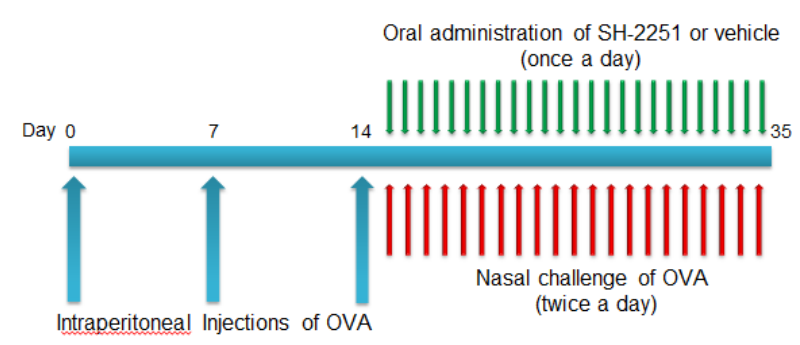

Figure 1: Design of an experiment for assessing allergic rhinitis in a murine model. A schematic representation of the experimental schedule is shown here.

A B
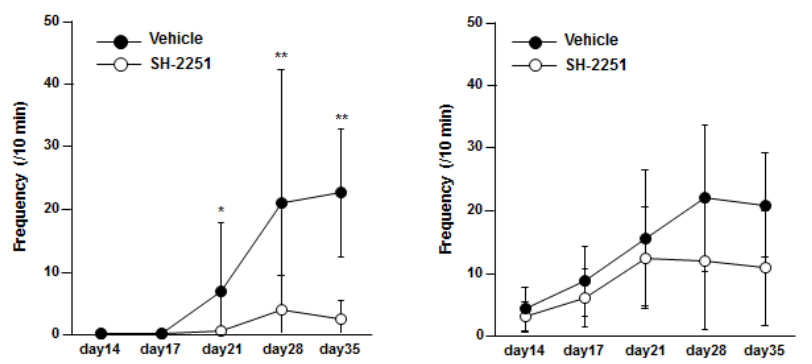

Figure 2: The effect of SH-2251 administration on the nasal symptoms of allergic rhinitis. The numbers of sneezing (A) and nasal rubbing (B) events within $10 \mathrm{~min}$ after the last intranasal OVA-challenge are indicated. Nasal symptoms were evaluated on days $14,17,21,28$ and 35 (mean $\pm \mathrm{SD}, \mathrm{n}=11$ per group). ${ }^{\star} \mathrm{P}<0.05$, ${ }^{*} \mathrm{P}<0.01$ (Student's t-test).

\section{SH-2251 suppressed the eosinophil infiltration and the swelling of the nasal mucosa in AR mice}

Next, we assessed the effect of SH-2251 administration on the infiltration of eosinophils and the swelling of the nasal mucosa in AR mice. Representative images of $\mathrm{H} \& \mathrm{E}$ staining (upper panel) and Giemsa staining (lower panel) of the nasal septum of SH-2251-treated or vehicle-treated mice on day 36 are shown in (Figures 3A). We conducted measurements at five different sites of the nasal septum, lateral walls and the nasal turbinate of bilateral nasal cavity to assess the effect of SH-2251 administration on eosinophil infiltration (Figure 3B left panel) and the thickness of the nasal mucosa (Figure 3B right panel). Both the eosinophil infiltration and the thickness of the nasal mucosa were significantly lower in the SH-2251-treated mice than in vehicle-treated control mice.

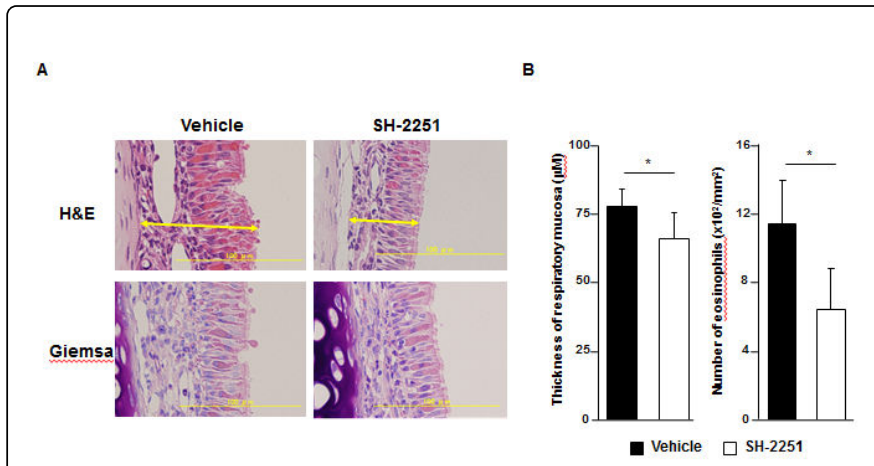

Figure 3: The effect of SH-2251 administration on the eosinophil infiltration and swelling of the nasal mucosa. (A) Representative images of $\mathrm{H} \& \mathrm{E}$ (upper) and May-Giemsa stains of the nasal septum of SH-2251- or vehicle-treated AR mice. (B) The number of infiltrated eosinophils in the nasal mucosa (left) and thickness of the nasal (right) are shown (mean $\pm \mathrm{SD}, \mathrm{n}=6$ per group). ${ }^{\star} \mathrm{P}<0.05$, ${ }^{* *} \mathrm{P}<0.01$ (Student's t-test).

\section{The expression of Il5 and Il13 mRNA at the nasal mucosa of AR mice was reduced by $\mathrm{SH}-2251$ administration}

Next, we assessed the effect of SH-2251 administration on the Th2 cytokine mRNA expression at the nasal mucosa in AR mice by qRTPCR. We previously reported that the differentiation of IL-5-producing Th2 cells and IL-5 production was inhibited by SH-2251 treatment [10]. As expected, the expression of Il5 mRNA in the nasal mucosa of the SH-2251-treaated group was significantly decreased compared to the vehicle group (Figure 4). Furthermore, the Il13 mRNA level was also decreased by SH-2251 administration, whereas the Il4 mRNA level was moderately increased (Figure 4). The expression of Ifn $\gamma$ mRNA was not affected by SH-2251 administration.
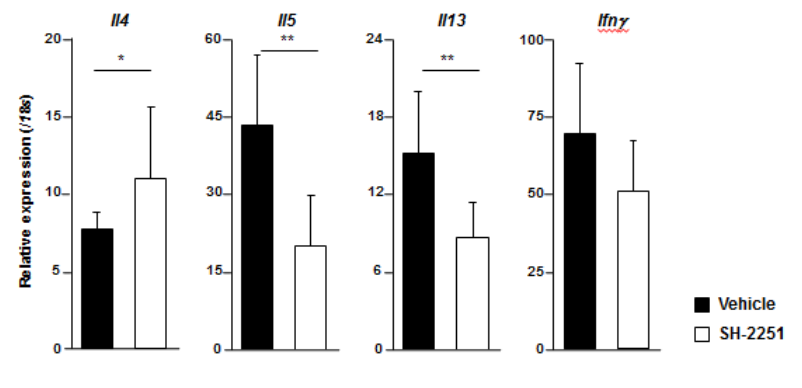

Figure 4: SH-2251 inhibits the Il5 and Il13 mRNA expression in the nasal mucosa of AR mice. The expression of Th2 cytokine (Il4, Il5, Il13) and Ifn $\gamma$ mRNA in the nasal mucosa was determined by qRTPCR. The results are shown as the relative intensity with ribosomal 18s RNA (mean $\pm \mathrm{SD}, \mathrm{n}=11$ per group). ${ }^{*} \mathrm{P}<0.01$ (Student's t-test).

\section{SH-2251 inhibited the expressions of Il1rl1 mRNA}

Recently, it was reported that IL-33 plays an important role in the pathogenesis of eosinophilic inflammation including AR $[13,14]$. Therefore, we next assessed the effect of SH-2251 on the expression of the IL-33 receptor. The receptor for IL-33 is composed of two proteins: 
IL-1rl1 and the IL-1r accessory protein (Il1rap) [15]. We found that the expression of Il1rl1 mRNA in the nasal mucosa of SH-2251-treated mice was significantly decreased compared to the vehicle group (Figure 5A). To confirm the inhibitory effect of SH-2251 on Illrl1 mRNA expression, we cultured splenic naïve CD4 T cells under Th2 conditions in the presence or absence of SH-2251 for 5 days, and mRNA expression was determined by as shown in Figure $5 \mathrm{~B}$. The expression of Il1rl1 mRNA in Th2 cells was inhibited by SH-2251 treatment. In sharp contrast, the expression of Il1rap was unaffected by SH-2251 treatment.
A

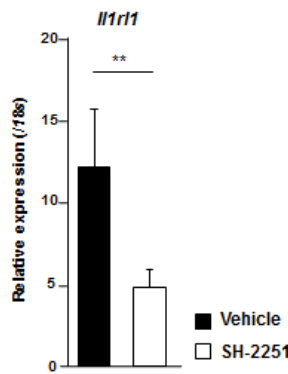

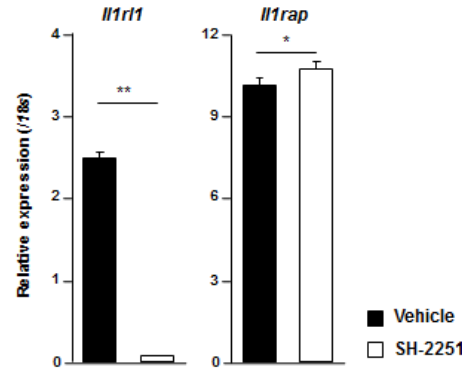

Figure 5: SH-2251 inhibits the Il1rl1 mRNA expression in vivo and in vitro. (A) The expression of Illrll mRNA in the nasal mucosa was determined by qRT-PCR. The results are shown as the relative intensity with $18 \mathrm{~s}$ ribosomal RNA (mean $\pm \mathrm{SD}, \mathrm{n}=11$ per group). ${ }^{* *} \mathrm{p}<0.01$ (Student's t-test). (B) The expression of Il1rl1 mRNA in in vitro differentiated Th2 cells was determined by qRT-PCR. Splenic naïve CD4 T cells were prepared and cultured under Th2 conditions for 5 days in the presence or absence of SH-2251 (100 nM). The results are shown as the relative intensity with 18 s ribosomal RNA (mean $\pm \mathrm{SD}, \mathrm{n}=3) .{ }^{*} \mathrm{P}<0.01$ (Student's t-test)

\section{Discussion}

It has been proposed that the symptoms of AR, such as rhinorrhea, sneezing and nasal obstruction, follow an early-phase reaction caused by histamine induced from mainly by mast cells in the first few minutes after antigen exposure. In addition, at more than $6 \mathrm{~h}$ after the challenge, nasal obstruction following swelling of the nasal mucosa results from the late-phase reaction caused by Th2 cytokines, various chemical mediators and chemokines [1]. In the present study, sneezing and nasal rubbing right after the last nasal challenge were attenuated by the administration of SH-2251. The reduced number of sneezing and nasal rubbing events indicates mitigation of the early-phase reaction. In addition, the infiltration of eosinophils and swelling of the nasal mucosa at $24 \mathrm{~h}$ later were also attenuated by the administration of SH-2251. The improvement of these histological findings indicates that the late-phase reaction was also mitigated. These results suggest that the administration of SH-2251 attenuates not only the early-phase reaction but also the late-phase reaction in AR.

Targeting the IL-5 seems to be the most obvious approach to selectively inhibiting eosinophilic inflammation, since IL-5 is the key cytokine responsible for the maturation, recruitment, activation and degranulation of eosinophils [16]. Humanized neutralizing monoclonal antibodies against IL-5 have been developed for the treatment of severe eosinophilic asthma [17]. Mepolizumab, a humanized monoclonal antibody against IL-5, showed a significant reduction in the annualized exacerbation rate compared with the placebo group in severe asthmatic patients [18]. In addition, Mepolizumab showed safe glucocorticoid-sparing effects in patients with oral steroid-dependent asthma [19]. These promising results were confirmed in large phase III trials [20-22]. In this study, we demonstrated the inhibitory effect of SH-2251 on the Il5 expression and eosinophilic infiltration in the mucosa of AR mice. Therefore, it is possible that SH-2251 has safe glucocorticoid-sparing effects in eosinophilic allergy patients.

Recently, the important role of mucosal epithelial cell-derived cytokine such as IL-25 and IL-33 in the induction of type 2 immune diseases has been reported $[23,24)$. Eosinophilic chronic rhinosinusitis (ECRS) was summarized as an entity of intractable chronic sinus inflammation accompanied by the infiltration of numerous activated eosinophils in the paranasal sinus mucosa and/or nasal polyps [25,26]. Several reports have shown that IL-33 plays a key role in the development of ECRS [27,28). In addition, the involvement of pathogenic Th2 (Tpath2) cells in the pathogenesis of ECRS was proposed $[28,29]$. Tpath2 cells are characterized by the expression of IL-33 receptor (Il1rl1) and ability to produceIL-5 [29]. We demonstrated that the expression of Il1rl1 was also decreased by the administration of SH-2251. Therefore, it is possible that SH-2251 may inhibit the generation of Tpath2 by inhibiting Illrl1 and Il5 expression.

Taken together, our current findings suggest that SH-2251 may be a new therapeutic candidate for not only AR but also severe eosinophilic inflammatory diseases.

\section{Author Contribution}

T.K. performed the experiments, analyzed the data and wrote the manuscript; S.M., K.A., H.O., Y.A. and Y.H. performed and supported the experiments; H.N. and M.Y. conceptualized the research, directed the study and edited the manuscript.

\section{Acknowledgements}

The authors would like to thank Mr. Takeshi Kiyoi for his excellent technical assistance and Ishihara Sangyo Kaisha, Ltd. for providing SH-2251. This work was supported by the JSPS KAKENHI Grant Numbers 816K191580, 917H040860 and 25860376, the Mochida Memorial Foundation for Medical and Pharmaceutical Research, the Takeda Science Foundation, the Uehara Memorial Foundation and the Naito Foundation.

\section{References}

1. Brozek JL, Bousquet J, Agache I, Agarwal A, Bachert C, et al. (2017) Allergic Rhinitis and its Impact on Asthma (ARIA) guidelines-2016 revision. J Allergy Clin Immunol 140: 950-958.

2. Pawankar R, Mori S, Ozu C, Kimura S (2011) Overview on the pathomechanisms of allergic rhinitis. Asia Pac Allergy 1: 157-167.

3. Okubo K, Kurono Y, Ichimura K, Enomoto T, Okamoto Y, et al. (2017) Japanese guidelines for allergic rhinitis 2017. Allergol Int 66: 205-219.

4. O'Shea JJ, Paul WE (2010) Mechanisms underlying lineage commitment and plasticity of helper CD4+ T cells. Science 327: 1098-1102.

5. Zhu J, Yamane H, Paul WE (2010) Differentiation of effector CD4 T cell populations $\left.{ }^{*}\right)$. Annu Rev Immunol 28: 445-489.

6. Sakaguchi S, Yamaguchi T, Nomura T, Ono M (2008) Regulatory T cells and immune tolerance. Cell 133: 775-787. 
Citation: Kitani T, Maruyama S, Aoishi K, Nishida N, Ogawa H, et al. (2017) Suppressive Effect of a Thioamide-Related Compound SH-2251 on a Murine Allergic Rhinitis Model. J Allergy Ther 8: 267. doi:10.4172/2155-6121.1000267

Page 5 of 5

7. Korn T, Bettelli E, Oukka M, Kuchroo VK (2009) IL-17 and Th17 Cells Annu Rev Immunol 27: 485-517.

8. Zhu J, Paul WE (2008) CD4 T cells: fates, functions, and faults. Blood 112: 1557-1569.

9. Wynn TA (2015) Type 2 cytokines: mechanisms and therapeutic strategies. Nat Rev Immunol 15: 271-282.

10. Suzuki J, Kuwahara M, Tofukuji S, Imamura M, Kato F, et al. (2013) A novel small compound SH-2251 suppresses Th2 cell-dependent airway inflammation through selective modulation of chromatin status at the Il5 gene locus. PLoS One 8: e61785.

11. Shinnakasu R, Yamashita M, Kuwahara M, Hosokawa H, Hasegawa A, et al. (2008) Gfi1-mediated stabilization of GATA3 protein is required for Th2 cell differentiation. J Biol Chem 283: 28216-28225.

12. Aoishi K, Takahashi H, Hato N, Gyo K, Yokota M, et al. (2016) Treatment of allergic rhinitis with intranasal infusion of botulinum toxin type A in mice. Life Sci 147: 132-136.

13. Akasaki S, Matsushita K, Kato Y, Fukuoka A, Iwasaki N, et al. (2016) Murine allergic rhinitis and nasal Th2 activation are mediated via TSLP and IL-33-signaling pathways. Int Immunol 28: 65-76.

14. Nakanishi W, Yamaguchi S, Matsuda A, Suzukawa M, Shibui A, et al. (2013) IL-33, but not IL-25, is crucial for the development of house dust mite antigen-induced allergic rhinitis. PLoS One 8: e78099.

15. Liew FY, Girard JP, Turnquist HR (2016) Interleukin-33 in health and disease. Nat Rev Immunol 16: 676-689.

16. Yanagibashi T, Satoh M, Nagai Y, Koike M, Takatsu K (2017) Allergic diseases: From bench to clinic - Contribution of the discovery of interleukin-5. Cytokine 98: 59-70.

17. Bel EH, Ten Brinke A (2017) New Anti-Eosinophil Drugs for Asthma and COPD: Targeting the Trait! Chest S0012-3692.

18. Haldar P, Brightling CE, Hargadon B, Gupta S, Monteiro W, et al. (2009) Mepolizumab and exacerbations of refractory eosinophilic asthma. $\mathrm{N}$ Engl J Med 360: 973-984.
19. Nair P, Pizzichini MM, Kjarsgaard M, Inman MD, Efthimiadis A, et al. (2009) Mepolizumab for prednisone-dependent asthma with sputum eosinophilia. N Engl J Med 360: 985-993.

20. Pavord ID, Korn S, Howarth P, Bleecker ER, Buhl R, et al. (2012) Mepolizumab for severe eosinophilic asthma (DREAM): a multicentre, double-blind, placebo-controlled trial. Lancet 380: 651-659.

21. Ortega HG, Liu MC, Pavord ID, Brusselle GG, FitzGerald JM, et al. (2014) Mepolizumab treatment in patients with severe eosinophilic asthma. N Engl J Med 371: 1198-1207.

22. Bel EH, Wenzel SE, Thompson PJ, Prazma CM, Keene ON, et al. (2014) Oral glucocorticoid-sparing effect of mepolizumab in eosinophilic asthma. N Engl J Med 371: 1189-1197.

23. Kubo M (2017) Innate and adaptive type 2 immunity in lung allergic inflammation. Immunol Rev 278: 162-172.

24. Joulia R, L'Faqihi FE, Valitutti S, Espinosa E (2017) IL-33 fine tunes mast cell degranulation and chemokine production at the single-cell level. J Allergy Clin Immunol 140: 497-509 e10.

25. Garg A, Das-Bhaumik R, Nesbitt AD, Levene AP, Joshi N, et al. (2010) Visual loss secondary to eosinophilic mucin rhinosinusitis in a woman: a case report. J Med Case Rep 4: 350.

26. Ferguson BJ (2000) Eosinophilic mucin rhinosinusitis: a distinct clinicopathological entity. Laryngoscope 110: 799-813.

27. Haenuki Y, Matsushita K, Futatsugi-Yumikura S, Ishii KJ, Kawagoe T, et al. (2012) A critical role of IL-33 in experimental allergic rhinitis. J Allergy Clin Immunol 130: 184-94 e11.

28. Endo Y, Hirahara K, Iinuma T, Shinoda K, Tumes DJ, et al. (2015) The interleukin-33-p38 kinase axis confers memory $\mathrm{T}$ helper 2 cell pathogenicity in the airway. Immunity 42: 294-308.

29. Shinoda K, Hirahara K, Nakayama T (2017) Maintenance of pathogenic Th2 cells in allergic disorders. Allergol Int 66: 369-376. 